\title{
Estrutura e composição florística de um Cerrado sensu stricto e sua importância para propostas de restauração ecológica
}

\author{
Paula Reys ${ }^{1}$, Maria Gabriela Gutierrez de Camargo ${ }^{1}$, Maria Tereza Grombone-Guaratini ${ }^{2,4}$, \\ Aloysio de Pádua Teixeira ${ }^{3}$, Marco Antonio Assis ${ }^{3}$ e Leonor Patricia Cerdeira Morellato ${ }^{3}$
}

Recebido: 1.08.2013; aceito: 12.08.2013

\begin{abstract}
Structure and floristic composition of a Cerrado sensu stricto and its relevance to ecological restauration). Edges are transition areas between two adjacent ecosystems that can be originated by natural or anthropogenic events. The edge influence includes both direct effects, related to changes on microenvironmental factors, as well as indirect effects, related to changes in species interactions. Additionally, some studies suggest that microenvironmental factors are altered by the cardinal orientation of the edge. The present study aimed to evaluate the influence of edge on microenvironmental factors, local soil conditions, woody vegetation structure, and species richness in a Cerrado sensu stricto at Itirapina, São Paulo State, Brazil. Specifically, we investigated whether microenvironmental factors (relative humidity (\%), photosynthetic active radiation (PAR), temperature and percentage of canopy cover), soil conditions and vegetation composition differ among plots ( 36 plots of $25 \times 2 \mathrm{~m}$ spaced $50 \mathrm{~m}$ apart) distributed on the edge and in the interior, facing east and south sides of the Cerrado. We sampled all woody trees and treelets with circumference $\geq 3 \mathrm{~cm}$ at $30 \mathrm{~cm}$ from its ground base within each plot. The microenvironmental variables relative humidity, PAR (photosynthetic active radiation), temperature and percent of canopy openness, and the soil samples $(0-20 \mathrm{~cm}$ deep $)$ for nutrients and texture analyses, were all collected at three points within each plot. We recorded a total of 120 woody species distributed among 40 plant families. Among the structural parameters of vegetation, only the absolute density was significantly higher in the interior south of the Cerrado. The microenvironmental factors were significantly different between the east and south sides, but did not differ between edge and interior. Therefore, based on the environmental and structural parameters we evaluated, there was no evidence of edge influence in the Cerrado studied. However, the east and south faces and associated microenvironmental factors, and the presence of some nutrients in the soil, such as $\mathrm{Mn}$, affected the Cerrado vegetation structure and species composition. Our results highlight the importance of taking into account the cardinal orientation in addition to the soil nutrient and microenvironment parameters to better understand the factors influencing the vegetation at a local scale.
\end{abstract}

Key words: diversity, edge influence, microclimate, savanna, similarity, species richness

RESUMO - (Estrutura e composição florística de um Cerrado sensu stricto e sua importância para propostas de restauração ecológica). Bordas são áreas de transição entre dois ecossistemas e podem ter origem natural ou antrópica. A influência de borda inclui tanto os efeitos diretos, relacionados às alterações microambientais, como os efeitos indiretos que afetam as interações entre espécies. Adicionalmente, estudos sugerem que a orientação cardinal pode modificar os efeitos microambientais da borda. O objetivo deste estudo foi avaliar a influência de borda sobre os fatores microambientais, as condições edáficas locais, a densidade e a riqueza de espécies em um Cerrado sensu stricto em Itirapina, SP, Brasil. Especificamente, nós investigamos se as características microambientais (umidade relativa do ar (\%), radiação fotossinteticamente ativa (PAR), temperatura e porcentagem de abertura de dossel), as condições edáficas e a composição da vegetação diferem entre parcelas ( 36 parcelas de $25 \times 2 \mathrm{~m}$ distanciadas $50 \mathrm{~m}$ entre si) distribuídas na borda e no interior, orientadas a leste e a sul do Cerrado estudado. Todos os indivíduos lenhosos com circunferência a $30 \mathrm{~cm}$ da base do caule $\geq 3 \mathrm{~cm}$ foram amostrados e marcados. As variáveis microambientais e as amostras de solo para análises químicas e granulométricas ( $0-20 \mathrm{~cm}$ de profundidade) foram coletadas em três pontos de cada parcela. Foi registrado um total de 120 espécies distribuídas em 40 famílias. Dentre os parâmetros estruturais da vegetação, somente a densidade absoluta foi significativamente maior no interior sul do fragmento. As variáveis microambientais diferiram significativamente entre as faces leste e sul, mas não entre borda e interior. Os parâmetros microambientais e estruturais não caracterizaram uma influência da borda. Entretanto, a orientação cardinal leste e sul e fatores microambientais associados, e a presença de determinados nutrientes no solo como o Mn, influenciaram a estrutura da vegetação de Cerrado e a sua composição em espécies. Nossos resultados ressaltam a importância de considerar a orientação cardinal, além da composição de nutrientes no solo, e dos fatores microambientais, para compreender as variações da vegetação em escala local. Palavras-chave: diversidade, influência de borda, microclima, savana, similaridade, riqueza de espécies

1. Universidade Estadual Paulista "Júlio de Mesquita Filho", Departamento de Botânica, Laboratório de Fenologia, Caixa Postal 199, 13506-900 Rio Claro, SP, Brasil

2. Instituto de Botânica, Núcleo de Pesquisa em Ecologia, Caixa Postal 68041, 04045-972 São Paulo, SP, Brasil

3. Universidade Estadual Paulista "Júlio de Mesquita Filho", Departamento de Botânica, Caixa Postal 199, 13506-900 Rio Claro, SP, Brasil

4. Autor para correspondência: mgromboneguaratini@gmail.com 


\section{Introdução}

O Cerrado, segunda maior província de vegetação do Brasil (Klink \& Machado 2005), é caracterizado por um gradiente vegetacional diversificado, que engloba a forma campestre aberta, como os campos limpos, até formas relativamente densas, florestais, como os cerradões (Coutinho 1978). Entre esses dois extremos fisionômicos, há uma gama de formas intermediárias, com fisionomia de savana, como os campos sujos, os campos cerrados, os cerrados sensu stricto (s.s.) (Coutinho 2006). De acordo com as condições locais de solo, topografia, água subterrânea e histórico de fogo, uma combinação dessas fisionomias pode aparecer em uma mancha de Cerrado, caracterizando um mosaico (Pivello et al. 1999).

Essa complexidade paisagística e ecológica dos Cerrados abriga uma rica biodiversidade, com altos números de espécies vasculares endêmicas (Bittencout \& Mendonça 2004, Klink \& Machado 2005), sendo considerado um "hotspot" de biodiversidade (Myers et al. 2000). Entretanto, a expansão da fronteira agrícola brasileira levou à redução de sua área de cobertura original e a altas taxas de extinção de espécies (Felfili \& Silva 1992). Atualmente, estima-se que apenas cerca de $2,2 \%$ da área de cobertura original dos Cerrados esteja sob proteção legal (Marris 2005). No Estado de São Paulo, essa vegetação que cobria, originalmente, cerca de $14 \%$ da área está atualmente reduzida a fragmentos que correspondem ao redor de 0,81\% (Kronka et al. 2005). A intensa fragmentação do Cerrado transformou a vegetação em manchas rodeadas por pastos e agricultura propiciando a criação de bordas que favorecem a invasão por gramíneas (Pivello et al. 1999), consideradas como um dos distúrbios que efetivamente ameaçam os remanescentes de Cerrado (Siqueira \& Durigan 2007).

Bordas são áreas de transição usualmente abrupta entre dois ecossistemas (Murcia 1995, Baker et al. 2002). Essas áreas podem ocorrer de forma natural em função de alterações edáficas, geomorfológicas e climáticas (Dangerfield et al. 2003) ou podem ser estabelecidas em função da fragmentação de origem antrópica (Murcia 1995). No último caso, as bordas delimitam o contato entre a vegetação nativa e uma área antrópica, como pastagens, áreas agrícolas ou urbanas (Murcia 1995). A influência de borda inclui efeitos diretos relacionados à diminuição da umidade, aumento de luz e temperatura e maior impacto dos ventos, variação na quantidade de nutrientes e matéria orgânica e efeitos indiretos que afetam as interações entre espécies (Murcia 1995, Kapos et al. 1997, Honnay et al. 2002, Toledo-Aceves \& García-Oliva 2008). Associado a esses fatores, é sugerido que a orientação cardinal pode modificar e mesmo intensificar os efeitos microclimáticos da borda (Chen et al. 1995, Camargo et al. 2011). Entretanto, efeitos de borda foram estabelecidas com base em estudos realizados em ecossistemas florestais (ver Laurance 2008, Kapos et al. 1997). Para os cerrados a influência de borda ainda não está estabelecida, uma vez que essa fitofisionomia é naturalmente mais aberta e as transições entre a vegetação e a matriz circundante usualmente não são tão abruptas.

A pesquisa ecológica realizada nos cerrados ao longo dos anos tem sido focada principalmente na avaliação da estrutura e florística (e.g. Oliveira Filho et al. 2002; Assunção \& Felfili 2004; Felfili et al. 2004; Fonseca \& Silva Júnior 2004; Balduino et al. 2005, Nettesheim et al. 2010); fitogeografia (Oliveira-Filho \& Ratter 2002; Siqueira \& Durigan 2007); estimativas de biomassa e estoque de carbono (Barbosa \& Fearnside 2004, 2005, Rezende et al. 2006, Ribeiro et al. 2011); estratégias de conservação da biodiversidade (Durigan et al. 2006); adaptações das plantas às condições dos solos de Cerrado (Araújo \& Haridasan 1988; Haridasan 2008); fenologia e ecologia reprodutiva (Gottsberger \& SilbebauerGottsberger 2006; Morellato et al. 2013). No entanto, trabalhos que investiguem a influência de borda no Cerrado são praticamente inexistentes (Lima-Ribeiro 2008, Jardim \& Batalha 2009; Camargo et al. 2011), embora o conhecimento florístico e estrutural da vegetação, bem como a avaliação da influência de borda sejam fundamentais para sua conservação e futuras propostas de manejo e recuperação dos remanescentes do Cerrado. Nesse contexto, considerando os atributos essenciais para a recuperação ecológica (Shackelford et al. 2013), o objetivos deste estudo foram avaliar a influência de borda sobre fatores microambientais, as condições edáficas locais, a densidade e a riqueza de espécies em áreas de borda e interior de um Cerrado sensu stricto no Estado de São Paulo. Especificamente nós investigamos se as características microambientais (umidade relativa do ar (\%), radiação fotossinteticamente ativa (PAR), temperatura e porcentagem de abertura de dossel), as condições edáficas e a composição da vegetação diferem entre parcelas distribuídas na borda e no interior, com orientação a leste e a sul, do Cerrado. 


\section{Material e métodos}

Área de estudo - O estudo foi realizado em uma área de Cerrado sensu stricto (ca. 260 ha), localizado no município de Itirapina $\left(22^{\circ} 13^{\prime} \mathrm{S}, 47^{\circ} 53^{\prime} \mathrm{W}\right)$, Estado de São Paulo. Esta área está incluída entre as prioritárias para a realização de inventário biológico no Estado de São Paulo (Durigan et al. 2006; Metzger \& Rodrigues 2008). O clima da região é do tipo Cwa segundo a classificação de Köppen (1948), ou seja, mesotérmico com invernos secos e verões chuvosos. As temperaturas médias mensais variam entre $18{ }^{\circ} \mathrm{C}$ (julho) e $32{ }^{\circ} \mathrm{C}$ (fevereiro) e a precipitação média anual é de $1.524 \mathrm{~mm}$ (Camargo et al. 2011).

O Cerrado sensu stricto estudado apresenta cobertura de copa descontínua, para o componente arbóreo dominante em torno de seis ou sete metros de altura e estrato herbáceo descontínuo. Em algumas áreas a cobertura é mais fechada, com vegetação mais densa e árvores mais desenvolvidas que alcançam 12 metros de altura. A área de estudo foi fragmentada há cerca de 30 anos e apresenta uma forma retangular com os lados voltados aos quatro pontos cardeais. As bordas/faces estudadas foram selecionadas por representarem a face mais escura (sul) e a mais iluminada (leste) do Cerrado; pela similaridade da matriz, predominantemente pastagem; e por não apresentarem distúrbios devido ao fogo, como o ocorrido na face norte há 20 anos, ou pela construção de rodovia na face oeste (Camargo et al. 2011).

Amostragem da comunidade - Foram estabelecidas 36 parcelas de $25 \times 2 \mathrm{~m}\left(50 \mathrm{~m}^{2}\right)$ distanciadas $50 \mathrm{~m}$ entre si, distribuídas nos quatro ambientes: borda leste (10 parcelas), borda sul (10), interior leste (8) e interior sul (8). Tanto na face leste quanto na face sul, as parcelas foram alocadas ao longo de duas linhas paralelas, sendo uma na borda - definida como a zona de contato com a matriz circundante, e outra distanciada $100 \mathrm{~m}$ em direção ao interior do remanescente. Em cada parcela, entre janeiro de 2004 a dezembro de 2006, todos os indivíduos lenhosos (arbóreos e arbustivos) vivos ou mortos em pé, com circunferência a $30 \mathrm{~cm}$ da base do caule $(\mathrm{CBC}) \geq 3 \mathrm{~cm}$, foram amostrados, plaqueados e tiveram a altura estimada com auxílio de vara graduada. Caules bifurcados ou múltiplos foram somados após o cálculo de área basal de cada caule, para que não houvesse superestimativa desse descritor. As espécies foram identificadas por meio de literatura, com o auxílio de especialistas e pela comparação com material depositado no Herbário
Rioclarense (HRCB), onde foram depositadas as exsicatas provenientes deste estudo. As espécies foram classificadas nas famílias reconhecidas pelo sistema Angiosperm Phylogeny Group III (APG 2009).

Variáveis microambientais - Medidas de umidade relativa do ar (\%), radiação fotossinteticamente ativa (PAR), temperatura e porcentagem de abertura de dossel foram registradas em três pontos equidistantes nas 36 parcelas, simultaneamente, em dias consecutivos, das 10 às 14 h nas estações seca e chuvosa. As medidas de temperatura, PAR e umidade relativa foram obtidas com o aparelho HOBO Micro Station (Onset Computer Corporation). Para avaliar possíveis diferenças na entrada de luz entre os ambientes foram registradas fotos hemisféricas a $1,30 \mathrm{~m}$ de altura do solo, com auxílio de máquina fotográfica digital COOLPIX 8700 e lentes hemisféricas NIKON FC-E9 ("olho de peixe"). As fotos digitalizadas foram analisadas pelos do programa GLA (Gap Light Analyzer - Frazer et al. 1999) que fornece estimativas sobre a porcentagem total de luminosidade média que atinge o solo. Para efeito das análises ambientais foi calculada a média por parcela.

Caracterização edáfica - Uma amostra de solo, composta por três subamostras retiradas ao acaso na profundidade de 0 a $20 \mathrm{~cm}$ com o auxílio de uma sonda, foi obtida em cada uma das 36 parcelas. As análises químicas e granulométricas foram realizadas na Universidade Federal de São Carlos (Araras, Estado de São Paulo) seguindo o procedimento proposto por Raij (1983). As propriedades químicas do solo obtidas foram $\mathrm{pH}$, matéria orgânica (M.O.), potássio (K), cálcio $(\mathrm{Ca})$, magnésio $(\mathrm{Mg})$, hidrogênio mais alumínio $(\mathrm{H}+\mathrm{Al})$, enxofre $(\mathrm{S})$, boro $(\mathrm{B})$, cobre $(\mathrm{Cu})$, ferro $(\mathrm{Fe})$, manganês $(\mathrm{Mn})$ e zinco $(\mathrm{Zn})$, além da soma de bases (SB), capacidade de troca catiônica (CTC) e saturação por bases (V), que expressam fertilidade do solo (sensu Reatto et al. 1998). Na análise granulométrica foram determinadas as porcentagens de argila, silte, areia grossa, areia fina e areia total.

Parâmetros estruturais - Foram calculadas a densidade relativa (DR), frequência relativa (FR), dominância relativa (DoR), valor de importância (VI) e valor de cobertura (VC) segundo Müeller-Dombois \& Ellenberg (1974), utilizando-se o programa FITOPAC (Shepherd 2010). A similaridade florística entre os ambientes (borda e interior, faces leste e sul) foi estimada pelo índice de Chao-Sørensen (Chao et al. 2005, 2006), que reduz o viés da sub amostragem 
por estimar e compensar a ocorrência de espécies compartilhadas e não amostradas. Esse índice foi escolhido, em detrimento ao índice clássico de Sørensen, porque possibilita avaliar a similaridade entre amostras de diferentes tamanhos (Chao et al. 2005, 2006). O índice de Chao-Sørensen foi calculado com intervalos de confiança (95\%) no programa EstimateS 8.2 (Colwell 2009). As curvas de rarefação foram construídas com $95 \%$ de Intervalo de Confiança (IC) usando a função $\mathrm{S}_{\text {obs }}$ (Mao Tau) (Cowell et al. 2004). Para comparar o número de espécies encontradas nos ambientes de borda e interior do Cerrado foi utilizado o programa EstimateS 8.2 (Colwell 2009), considerando a sobreposição dos IC como indicação da ausência de diferença.

Considerando que a premissa de normalidade não foi satisfeita para a realização de teste paramétrico, mesmo após a transformação dos dados, os valores médios de cada variável microambiental e edáfica entre ambientes (borda leste e sul e interior leste e sul) foram comparados por meio de análise de variância não paramétrica (Kruskal-Wallis), tendo como réplica cada parcela, seguidas de teste de Dunn (Zar 1999). O mesmo teste foi utilizado para comparar os descritores estruturais (área basal, densidades absoluta e relativa) entre os quatro ambientes (Zar 1999). Foi construído um gráfico onde os indivíduos foram distribuídos em classes de altura de dois metros, com indivíduos acima de 6 metros colocados em uma única classe por serem em menor número. A comparação entre as classes de altura e diâmetro entre os quatro ambientes, foi realizada pela ANOVA seguida de teste de Tukey (Zar 1999) tendo em vista que, nesses casos os parâmetros de normalidade e homocedasticidade foram satisfeitos. Posteriormente, os ambientes foram agrupados em leste e sul e em borda e interior e comparados pelo teste de Mann-Whitney (Zar 1999) seguida de teste de Dunn, tendo em vista que o parâmetro de normalidade não foi satisfeito.

Para produzir uma ordenação das parcelas a partir das variáveis microambientais e das propriedades físicas do solo efetuamos uma análise de componentes principais (PCA), sendo que as variáveis redundantes ou que pouco contribuíram foram eliminadas. Dessa forma, a matriz de covariância consistiu de três variáveis ambientais (temperatura, \% de abertura da copa e umidade relativa) e quatro propriedades físico-químicas do solo (argila, areia, $\mathrm{pH}$, matéria orgânica, alumínio e manganês). A análise foi realizada com matriz de covariância sendo que as variáveis expressas em proporção foram transformadas em $\log (\mathrm{x}+1)$ para compensar os desvios causados por valores muito elevados. A análise global das relações entre a distribuição da abundância de espécies e as variáveis microambientais e edáficas foram exploradas com a análise de correspondência canônica (CCA) (Ter-Braak 1987), de acordo com os critérios propostos por Legendre \& Legendre (1998), através do programa PC-ORD 6.0 para Windows (McCune $\&$ Mefford, 2000). A matriz principal consistiu no número de indivíduos por espécie (variáveis) por parcela (objeto), para os quais 59 espécies com 10 indivíduos ou mais foram consideradas; e a matriz secundária incluiu as variáveis microambientais e as propriedades químicas e físicas do solo (variáveis) por parcela. Primeiramente processamos a análise utilizando todas as variáveis e depois as variáveis multicolineares (correlações entre variáveis $\geq 0,7$ ) e as variáveis fracamente correlacionadas $(\mathrm{r}<0,5 \mathrm{com}$ os eixos 1 e 2 da CCA) foram excluídas (Legendre \& Legendre 1998). Testamos a hipótese de independência entre as matrizes de abundância específica e de dados microambientais e edáficos por meio do teste Mantel, com 1.000 permutações de Monte Carlo. O teste de permutações de Monte Carlo foi realizado para testar a significância estatística da contribuição de todas as variáveis ambientais e dos eixos canônicos (Ter Braak 1988).

\section{Resultados}

Foram amostrados 2.740 indivíduos nos quatro ambientes estudados, distribuídos em 120 espécies de 40 famílias; apenas uma família permaneceu indeterminada (tabela 1). As famílias com maior número de espécies foram Myrtaceae (15), Fabaceae (13), Malpighiaceae (9), Vochysiaceae (8) e Bignoniaceae (7). Cerca de 50\% das famílias (22) estiveram representadas por apenas uma espécie. As espécies mais abundantes foram Bauhinia rufa (Bong.) Steudel, predominantemente nas bordas, Xylopia aromatica (Lam.) Mart., Miconia rubiginosa (Bonpl.) A.DC., Virola sebifera Aubl. e Myrcia guianensis (Aubl.) DC. Dentre essas, Virola sebifera foi a única espécie não encontrada em todos os ambientes amostrados, sendo mais comum na borda sul, mais sombreada (tabela 1).

A densidade total estimada foi de 15.522 indivíduos por hectare. O maior diâmetro (DBC) obtido foi $34,7 \mathrm{~cm}$ e a altura máxima foi $12 \mathrm{~m}$. Foram encontradas diferenças significativas na altura $(\mathrm{F}=30,15 ; \mathrm{p}<0,0001, \mathrm{gl}=3)$ e no diâmetro 
Tabela 1. Famílias e espécies amostradas nos quatro ambientes em um Cerrado sensu stricto em Itirapina, SP, Brasil. Co: código da espécie; r: correlação de Pearson; BL: borda leste; BS: borda sul; IL interior leste; IS: interior sul; Ni: número de indivíduos amostrados. Asterisco (*) indica que a espécie foi usada na Análise de Correspondência Canônica (CCA).

Table 1.Families and species sampled in the four environments in a Cerrado sensu stricto at Itirapina, São Paulo State, Brazil. Co: species code; r: Pearson correlation; BL: East Edge; BS: South Edge; IL: East Interior; IS: South Interior; Ni: number of individuals. Asterisks $\left(^{*}\right)$ indicate that the species was used in the ordination analyses (CCA).

\begin{tabular}{|c|c|c|c|c|c|c|c|c|}
\hline Famílias & Espécies & Co & $\mathrm{r}$ & $\mathrm{BL}$ & BS & IL & IS & $\mathrm{Ni}$ \\
\hline \multirow[t]{3}{*}{ Annonaceae } & Annona coriacea Mart & & & & 1 & 4 & 4 & 9 \\
\hline & Duguetia furfuracea (A.St.-Hil) Saff & & & 1 & & 1 & 1 & 3 \\
\hline & Xylopia aromatica (Lam.) Mart. * & 59 & 0,26 & 37 & 37 & 36 & 54 & 164 \\
\hline \multirow[t]{2}{*}{ Apocynaceae } & Aspidosperma tomentosum Mart. * & 4 & 0,17 & 6 & 2 & 18 & 8 & 34 \\
\hline & Aspidosperma sp. & & & & & 1 & & 1 \\
\hline Araliaceae & $\begin{array}{l}\text { Schefflera vinosa (Cham. \& Schltdl) Frodin } \\
\text { \& Fiaschi * }\end{array}$ & 48 & $-0,41$ & 12 & 13 & 10 & 27 & 62 \\
\hline Arecaceae & Syagrus flexuosa (Mart.) Becc. & & & & 3 & & & 3 \\
\hline \multirow[t]{7}{*}{ Asteraceae } & Baccharis dracunculifolia DC. & & & & 1 & & & 1 \\
\hline & $\begin{array}{l}\text { Chromolaena laevigata (Lam.) R.M.King \& } \\
\text { H.Rob. }\end{array}$ & & & 2 & & 3 & 1 & 6 \\
\hline & Gochnatia barrosii Cabrera * & 22 & 0,44 & 4 & 5 & 2 & 5 & 16 \\
\hline & Gochnatia pulchra Cabrera * & 23 & 0,20 & 3 & & 6 & 3 & 12 \\
\hline & Piptocarpha rotundifolia Baker * & 42 & $-0,19$ & 4 & 1 & 5 & & 10 \\
\hline & Vernonia ferruginea Less. & & & 1 & 1 & 1 & & 3 \\
\hline & Vernonia sp. & & & 2 & & & & 2 \\
\hline \multirow[t]{6}{*}{ Bignoniaceae } & Arrabidaea brachypoda Bureau & & & 2 & & & 1 & 3 \\
\hline & Distictella elongata Urb. & & & 1 & & & & 1 \\
\hline & Jacaranda caroba (Vell.) A.DC. * & 25 & 0,14 & 6 & & 2 & 5 & 13 \\
\hline & Jacaranda rufa Silva Manso & & & & & 1 & & 1 \\
\hline & Memora axillaris K.Schum. * & 28 & $-0,01$ & 10 & 2 & 15 & 6 & 33 \\
\hline & Tabebuia ochracea (Cham.) Standl. * & 55 & 0,23 & 8 & & 8 & 1 & 17 \\
\hline Burseraceae & Protium heptaphyllum (Aubl.) Marchand & & & & 2 & & & 2 \\
\hline Caryocaraceae & Caryocar brasiliense A.St.-Hil. & & & & 2 & 3 & & 5 \\
\hline \multirow[t]{2}{*}{ Celastraceae } & Plenckia populnea Reissek & & & & & & 3 & 3 \\
\hline & $\begin{array}{l}\text { Peritassa campestris (Cambess. ex A.St.-Hil.) } \\
\text { A.C.Sm. }\end{array}$ & & & & & 2 & & 2 \\
\hline Chrysobalanaceae & Licania sp. & & & & 1 & & & 1 \\
\hline \multirow[t]{2}{*}{ Connaraceae } & Connarus suberosus Planch. * & 11 & $-0,15$ & 4 & 1 & 17 & 3 & 25 \\
\hline & Rourea induta Planch. * & 47 & 0,39 & 7 & 5 & 8 & 4 & 24 \\
\hline Dilleniaceae & Davilla elliptica A.St.-Hil. & & & 1 & 2 & & & 3 \\
\hline Ebenaceae & Diospyros hispida A.DC. * & 16 & $-0,13$ & 7 & 6 & 18 & 2 & 33 \\
\hline \multirow[t]{4}{*}{ Erythroxylaceae } & Erythroxylum cuneifolium (Mart.) O.E.Schulz* & 18 & $-0,04$ & 8 & 9 & 4 & 17 & 38 \\
\hline & Erythroxylum suberosum A.St.-Hil. * & 19 & 0,20 & 14 & 4 & 47 & 7 & 72 \\
\hline & Erythroxylum tortuosum Mart. * & 20 & $-0,27$ & 2 & 2 & 7 & 4 & 15 \\
\hline & Erythroxylum buxus Peyr. & & & & 2 & & 2 & 4 \\
\hline \multirow[t]{3}{*}{ Fabaceae } & $\begin{array}{l}\text { Anadenanthera peregrina var. falcata (Benth.) } \\
\text { Altschul. }\end{array}$ & 3 & $-0,03$ & 41 & 2 & 33 & 23 & 99 \\
\hline & Bauhinia rufa (Bong.) Steud. * & 6 & $-0,36$ & 62 & 21 & 68 & 16 & 167 \\
\hline & Bowdichia virgilioides Kunth * & 27 & $-0,01$ & 5 & & 4 & 1 & 10 \\
\hline
\end{tabular}


Tabela 1 (continuação)

\begin{tabular}{|c|c|c|c|c|c|c|c|c|}
\hline Famílias & Espécies & $\mathrm{Co}$ & $\mathrm{r}$ & $\mathrm{BL}$ & BS & $\mathrm{IL}$ & IS & $\mathrm{Ni}$ \\
\hline & Copaifera langsdorffii Desf. * & 12 & $-0,07$ & & 8 & 9 & 8 & 25 \\
\hline & Dalbergia miscolobium Benth. ${ }^{*}$ & 13 & 0,38 & 8 & 8 & 4 & 14 & 34 \\
\hline & Dimorphandra mollis Benth. * & 15 & 0,09 & 8 & 1 & 8 & 1 & 18 \\
\hline & Leptolobium dasycarpum Vogel & & & 1 & & 2 & & 3 \\
\hline & Machaerium brasiliense Vogel & & & 1 & 1 & 2 & & 4 \\
\hline & Machaerium acutifolium Vogel & & & 9 & 1 & 8 & 9 & 27 \\
\hline & Pterodon emarginatus Vogel $*$ & 41 & $-0,27$ & & 18 & & & 18 \\
\hline & Senna rugosa (G.Don) H.S.Irwin \& Barneby * & 49 & 0,38 & 15 & & 19 & & 34 \\
\hline & Stryphnodendron adstringens (Mart.) Coville * & 53 & $-0,09$ & 1 & & 5 & 2 & 8 \\
\hline & Stryphnodendron obovatum Benth. * & 52 & $-0,19$ & 16 & 14 & 28 & 4 & 62 \\
\hline Flacourtiaceae & Casearia sylvestris $\mathrm{Sw}$. & & & 6 & 1 & 1 & & 8 \\
\hline Indeterminada & Indeterminada & & & 1 & 2 & 2 & 2 & 7 \\
\hline Lacistemataceae & Lacistema hasslerianum Chodat & & & & 2 & & & 2 \\
\hline Lamiaceae & Aegiphila lhotzkiana Cham. * & 1 & $-0,36$ & 11 & & 3 & 2 & 16 \\
\hline \multirow[t]{3}{*}{ Lauraceae } & Ocotea corymbosa $\mathrm{Mez} *$ & 51 & $-0,14$ & & 2 & 1 & 10 & 13 \\
\hline & Ocotea pulchella Mart. * & 36 & 0,04 & 14 & 31 & 24 & 30 & 99 \\
\hline & Ocotea sp. & & & & & & 1 & 1 \\
\hline \multirow[t]{7}{*}{ Malpighiacae } & Banisteriopsis campestris (A.Juss.) Little * & 5 & $-0,14$ & 36 & 2 & 33 & 8 & 79 \\
\hline & Banisteriopsis stellaris (Griseb.) B.Gates & & & & 2 & 2 & & 4 \\
\hline & Banisteriopsis sp. & & & & & & 1 & 1 \\
\hline & Byrsonima basiloba A.Juss. * & 7 & $-0,35$ & 8 & 2 & 11 & 2 & 23 \\
\hline & Byrsonima coccolobifolia Kunth * & 8 & $-0,17$ & 8 & & 13 & 2 & 23 \\
\hline & Byrsonima crassifolia (L.) Kunth & & & & 2 & & 1 & 3 \\
\hline & Byrsonima intermedia A.Juss. * & 9 & $-0,19$ & 6 & 10 & 5 & 22 & 43 \\
\hline Malvaceae & Eriotheca gracilipes (K.Schum.) A.Robyns * & 17 & 0,02 & 7 & 1 & 8 & 3 & 19 \\
\hline \multirow[t]{6}{*}{ Melastomataceae } & Leandra solenifera Cogn. * & 14 & 0,06 & 1 & 1 & 7 & 4 & 13 \\
\hline & Miconia albicans (Sw.) Triana * & 29 & $-0,15$ & 6 & 10 & 21 & 27 & 64 \\
\hline & Miconia ligustroides Naudin * & 30 & 0,62 & 2 & 2 & 5 & 2 & 11 \\
\hline & Miconia rubiginosa (Bonpl.) A.DC. * & 31 & 0,19 & 8 & 51 & 27 & 25 & 111 \\
\hline & Miconia fallax DC. * & 32 & 0,25 & 2 & 9 & 7 & 6 & 24 \\
\hline & Miconia sp. & & & & 1 & & & 1 \\
\hline \multirow[t]{4}{*}{ Moraceae } & Ficus citrifolia Mill. & & & 1 & 2 & & & 3 \\
\hline & Brosimum gaudichaudii Trécul & & & & & & 1 & 1 \\
\hline & Ficus citrifolia Mill. & & & 1 & 2 & & & 3 \\
\hline & Brosimum gaudichaudii Trécul & & & & & & 1 & 1 \\
\hline Myristicaceae & Virola sebifera Aubl. * & 57 & 0,27 & & 17 & 39 & 89 & 145 \\
\hline \multirow[t]{5}{*}{ Myrtaceae } & Blepharocalyx salicifolius (Kunth) O.Berg & & & 6 & & & 1 & 7 \\
\hline & $\begin{array}{l}\text { Campomanesia pubescens (Mart. ex DC.) } \\
\text { O.Berg }\end{array}$ & & & 12 & 9 & 30 & 8 & 59 \\
\hline & Eugenia bimarginata DC. * & 21 & 0,24 & 1 & 9 & 3 & 11 & 24 \\
\hline & Eugenia punicifolia (Kunth) DC. & & & & 1 & 2 & 2 & 5 \\
\hline & Eugenia pyriformis Cambess. & & & 2 & 2 & 2 & & 6 \\
\hline
\end{tabular}


Tabela 1 (continuação)

\begin{tabular}{|c|c|c|c|c|c|c|c|c|}
\hline Famílias & Espécies & $\mathrm{Co}$ & $\mathrm{r}$ & $\mathrm{BL}$ & $\mathrm{BS}$ & $\mathrm{IL}$ & IS & $\mathrm{Ni}$ \\
\hline & Myrcia bella Cambess. * & 33 & 0,12 & 16 & 2 & 29 & 10 & 57 \\
\hline & Myrcia fallax (Rich.) DC. * & 34 & $-0,25$ & 2 & 30 & 11 & 36 & 79 \\
\hline & Myrcia guianensis (Aubl.) DC. * & 35 & 0,53 & 40 & 19 & 44 & 37 & 140 \\
\hline & Myrcia splendens (Sw.) DC. * & 10 & 0,29 & 2 & 24 & 12 & 38 & 76 \\
\hline & Myrcia tomentosa (Aubl.) DC. & & & & 2 & & & 3 \\
\hline & Myrcia venulosa DC. & & & & 1 & & 3 & 4 \\
\hline & Myrcia sp. & & & & 1 & & 2 & 3 \\
\hline & Psidium cinereum Mart. ex DC. & & & 2 & & & 3 & 5 \\
\hline & Psidium grandifolium Mart. ex DC. & & & & & & 2 & 2 \\
\hline & Siphoneugena widgreniana O.Berg & & & & & & 1 & 1 \\
\hline \multirow[t]{2}{*}{ Nyctaginaceae } & Guapira noxia (Netto) Lundell * & 24 & 0,04 & 13 & 8 & 5 & 6 & 32 \\
\hline & Guapira opposita (Vell.) Reitz * & 40 & $-0,36$ & 3 & 4 & 1 & 3 & 11 \\
\hline Ochnaceae & Ouratea spectabilis Engl. * & 37 & 0,40 & 17 & 6 & 11 & 6 & 40 \\
\hline Peraceae & Pera obovata (Klotzsch) Baill. & & & & & & 1 & 1 \\
\hline Polygalaceae & Bredemeyera floribunda Willd. & & & & 2 & & 3 & 5 \\
\hline \multirow[t]{3}{*}{ Primulaceae } & Myrsine guianensis (Aubl.) Kuntze * & 44 & 0,28 & & & 4 & 16 & 20 \\
\hline & Myrsine umbellata Mart. * & 45 & 0,48 & & 2 & 1 & 10 & 13 \\
\hline & Myrsine sp. & & & & & & 1 & 1 \\
\hline Proteaceae & Roupala montana Aubl. * & 46 & $-0,06$ & & 6 & & 11 & 17 \\
\hline \multirow[t]{4}{*}{ Rubiaceae } & Amaioua guianensis Aubl. * & 2 & 0,55 & & 3 & 1 & 27 & 31 \\
\hline & Cordiera sessilis (Vell.) Kuntze & & & 1 & & 1 & & 2 \\
\hline & Palicourea rigida Kunth & & & 5 & & 3 & & \\
\hline & Tocoyena formosa (Cham. \& Schltdl.) K.Schum. * & 56 & 0,58 & 22 & 5 & 29 & 23 & 79 \\
\hline \multirow[t]{2}{*}{ Sapotaceae } & Pouteria ramiflora (Mart.) Radlk. * & 38 & $-0,21$ & 9 & 1 & 9 & & 19 \\
\hline & Pouteria torta (Mart.) Radlk. * & 39 & $-0,19$ & 20 & & 3 & 1 & 24 \\
\hline Siparunaceae & Siparuna guianensis Aubl. * & 50 & $-0,90$ & 3 & 14 & & 5 & 22 \\
\hline Solanaceae & Cestrum sp. & & & & & 1 & & \\
\hline Styracaceae & Styrax ferrugineus Nees \& Mart. * & 54 & $-0,36$ & 9 & 1 & 12 & 3 & 25 \\
\hline Verbenaceae & Lippia balansae Briq. * & 26 & 0,05 & 6 & 5 & 1 & 1 & 13 \\
\hline \multirow[t]{8}{*}{ Vochysiaceae } & Qualea dichotoma Warm. ex Wille & & & & 1 & & & 1 \\
\hline & Qualea grandiflora Mart. * & 43 & 0,32 & 12 & 12 & 9 & 7 & 40 \\
\hline & Qualea multiflora Mart. & & & & & 1 & & 1 \\
\hline & Qualea sp. & & & & 1 & & & 1 \\
\hline & Vochysia parviflora Spruce ex Warm. & & & 1 & & & & 1 \\
\hline & Vochysia tucanorum Mart. * & 58 & 0,38 & & 12 & & 12 & 24 \\
\hline & Vochysia sp. 1 & & & & & & 3 & 3 \\
\hline & Vochysia sp. 2 & & & & & & 6 & 6 \\
\hline
\end{tabular}

$(\mathrm{F}=20,26 ; \mathrm{p}<0,0001 ; \mathrm{gl}=3)$ das árvores entre os ambientes estudados. Entretanto, a borda leste e o interior leste não diferiram significativamente em altura; e o interior leste e o interior sul não diferiram em diâmetro $(p<0,05)$. No interior leste $70 \%$ dos indivíduos foram encontrados na menor classe de altura (figura 1a). No interior sul 64,7\% das árvores pertencem a menor classe de diâmetro (figura 1b).
Os parâmetros estruturais das 10 espécies mais abundantes estão listados na tabela 2. Somente a densidade absoluta foi significativamente maior no interior sul do Cerrado estudado $(\mathrm{F}=20,2654$; $\mathrm{p}=0,001)$. Os demais descritores florísticos e estruturais não diferiram entre os ambientes. Enquanto algumas espécies como Anadenanthera peregrina var. falcata (Benth.) Altschul., Myrcia guianensis e Xylopia 
aromatica apresentaram altos VI em todos ou na maior parte dos ambientes, outras espécies se destacaram-se apenas nos ambientes de determinada face, como Banisteriopsis campestris e Stryphnodendron obovatum Benth. na face leste e Virola sebifera Aubl. na face sul (tabela 1). Entretanto, nenhuma espécie apresentou VI destacado apenas na borda ou no interior, independentemente da face.

A riqueza de espécies foi semelhante entre borda (80 espécies) e interior (81) (figura 2). A similaridade foi maior entre as parcelas orientadas a leste $(0,77 \pm 0,03)$ e as parcelas orientadas a sul $(0,7 \pm 0,04)$ (tabela 3$)$.

As principais diferenças nas características microambientais foram observadas entre a face leste e sul do fragmento, enquanto poucas diferenças significativas foram encontradas entre as variáveis analisadas na borda e interior (tabela 4). De modo geral, a face leste foi significativamente mais iluminada, mais quente, com maior abertura de dossel e com menor umidade relativa do ar. Quanto às características edáficas, essa face apresentou solos
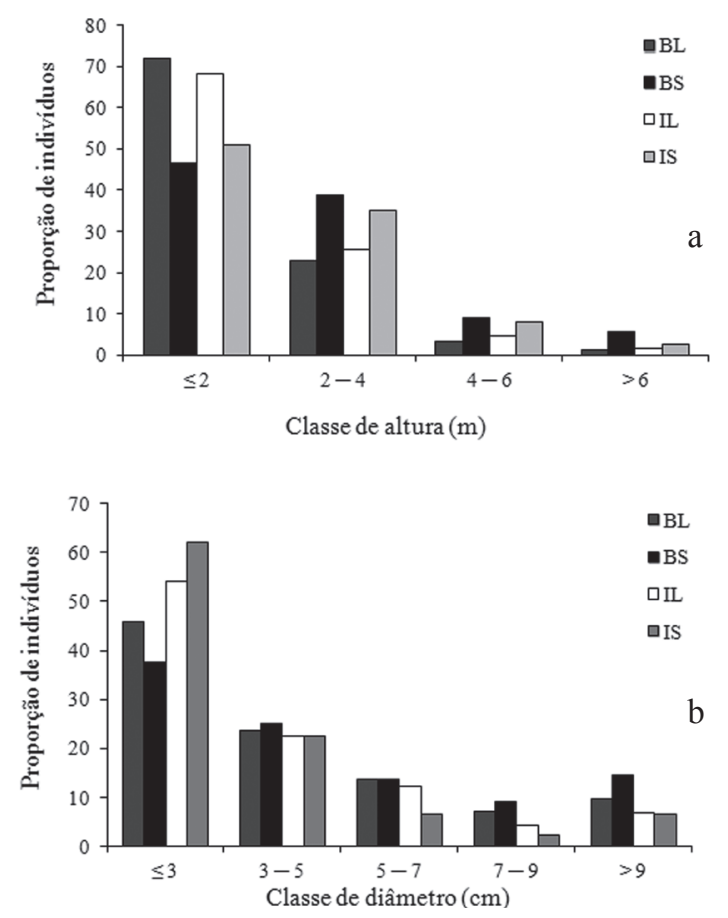

Figura 1. Proporção de indivíduos nas classes de altura (a) e de diâmetro (b) amostrados nos quatro ambientes de um Cerrado sensu stricto em Itirapina, SP, Brasil, BL: borda leste; BS: borda sul; IL: interior leste; IS: interior sul.

Figure 1. Proportion of indiviuals by height class (a) and diameter class (b) sampled in the four environments of a Cerrado sensu stricto at Itirapina, São Paulo State, Brazil, BL: East edge, BS: South edge; IL: East interior; IS: South interior. significativamente mais arenosos, menos ácidos, com menor porcentagem de matéria orgânica e menor CTC.

O diagrama de ordenação resultante da análise de componentes principais (PCA) das variáveis microambientais e de solo resumiu $73,8 \%$ da variabilidade total dos dados nos eixos 1 e 2 (figura 3). O diagrama eixo 1 mostrou uma tendência à formação de gradiente entre os ambientes (borda leste, interior leste, borda sul, interior sul) em função da porcentagem de abertura da copa, sendo que, a borda leste por apresentar maior grau de abertura da copa permaneceu isolada dos demais ambientes no lado positivo deste eixo. No lado positivo do eixo 1 foram ordenadas todas as parcelas da borda leste e metade das parcelas localizadas na borda sul em função da porcentagem de abertura da copa e de valores mais altos de $\mathrm{pH}$ do solo. Os valores de $\%$ de abertura da copa e $\mathrm{pH}$ tiveram alta correlação com o eixo 1 ( $r=0,94$ e $r=0,7$ respectivamente). No lado negativo do eixo 1 foram ordenadas todas as parcelas do interior sul e leste correlacionadas com uma menor porcentagem de abertura da copa e uma maior concentração de matéria orgânica $(\mathrm{MO})$ e alumínio (Al) no solo (figura 3). Os valores de concentração de $\mathrm{MO}$ e Al tiveram alta correlação negativa com o eixo 1 $(\mathrm{r}=-0,8$ para ambas as variáveis). No lado negativo do eixo 2 foram ordenadas todas as unidades amostrais da borda sul correlacionadas com maior quantidade de manganês $(\mathrm{Mn})$ e magnésio $(\mathrm{Mg})$. Os valores da correlação foram ( $r=-0,95$ e $r=-0,7$ respectivamente $)$. Este eixo mostra a formação de um gradiente sem a constituição de grupos coesos.

A análise de correspondência canônica (CCA) realizada com 59 espécies resumiu $23,6 \%$ da variabilidade total dos dados nos primeiros dois eixos (figura 4 a e b). A correlação de Pearson para os dois eixos $(r=0,8$ e $r=0,75)$ indicou uma forte relação entre a distribuição das espécies e as variáveis microambientais, sendo que o teste de permutação de Monte Carlo foi significativo para os eixos 1 e $2(p=0,002)$. De acordo com o coeficiente canônico, as variáveis de maior peso na ordenação foram a $\%$ de abertura da copa e a presença de $\mathrm{Al}$, os quais tiveram alta correlação com o eixo $1(\mathrm{r}=-0,9$ e $r=0,8$ respectivamente). O diagrama da CCA distinguiu dois grupos de parcelas (figura 4a). No lado negativo do eixo 1 estão as parcelas orientadas a leste, associadas à maior abertura do dossel e maior $\mathrm{pH}$, e no lado positivo, as parcelas orientadas a sul associadas à maior quantidade de alumínio e matéria orgânica. O eixo 2 segregou duas parcelas (BS11 e BS20) localizadas na borda sul do fragmento, que 
Tabela 2. Valores de Densidade absoluta (DA), densidade relativa (DR), frequência relativa (FR), dominância relativa (DoR), e valor de importância (VI) para as 10 espécies com maiores VIs nos quatro ambientes estudados no Cerrado sensu stricto de Itirapina, SP, Brasil.

Table 2. Absolute density (DA), relative density (DR), relative frequency (FR), relative dominance (DoR), and importance value (VI) for the 10 species with the highest VIs in each of the four environments studied in the Cerrado sensu stricto at Itirapina, São Paulo State, Brazil.

\begin{tabular}{|c|c|c|c|c|c|}
\hline \multirow[t]{2}{*}{ Espécie } & \multicolumn{5}{|c|}{ Parâmetros estruturais } \\
\hline & DA & DR & FR & DoR & VI \\
\hline \multicolumn{6}{|l|}{ Borda Leste } \\
\hline Anadenanthera peregrina & 820 & 6,48 & 2,95 & 10,65 & 6,70 \\
\hline Bauhinia rufa & 1.240 & 9,79 & 3,69 & 2,66 & 5,38 \\
\hline Myrcia guianensis & 800 & 6,32 & 3,69 & 4,77 & 4,93 \\
\hline Xylopia aromatica & 740 & 5,85 & 3,32 & 5,41 & 4,86 \\
\hline Pouteria torta & 400 & 3,16 & 1,48 & 6,23 & 3,62 \\
\hline Banisteriopsis campestris & 720 & 5,59 & 3,32 & 1,32 & 3,44 \\
\hline Qualea grandiflora & 240 & 1,90 & 2,21 & 5,18 & 3,10 \\
\hline Stryphnodendron obovatum & 320 & 2,53 & 2,58 & 3,76 & 2,95 \\
\hline Guapira noxia & 260 & 2,05 & 1,85 & 4,29 & 2,73 \\
\hline Aegiphila lhotszkyana & 220 & 1,74 & 2,21 & 4,00 & 2,65 \\
\hline \multicolumn{6}{|l|}{ Interior Leste } \\
\hline Anadenanthera peregrina & 825 & 3,91 & 2,86 & 21,70 & 6,82 \\
\hline Myrcia guianensis & 1.100 & 5,22 & 1,79 & 7,25 & 4,75 \\
\hline Bauhinia rufa & 1.700 & 8,07 & 2,50 & 3,65 & 4,74 \\
\hline Erythroxylum suberosum & 1.175 & 5,58 & 2,15 & 4,18 & 3,96 \\
\hline Xylopia aromatica & 900 & 4,27 & 2,86 & 4,61 & 3,91 \\
\hline Myrcia bella & 725 & 3,44 & 2,86 & 2,44 & 2,91 \\
\hline Miconia rubiginosa & 675 & 3,20 & 1,43 & 3,81 & 2,81 \\
\hline Ocotea pulchella & 600 & 2,85 & 0,71 & 4,28 & 2,61 \\
\hline Stryphnodendron obovatum & 700 & 3,32 & 2,15 & 1,97 & 2,48 \\
\hline Banisteriopsis campestris & 825 & 3,91 & 2,51 & 0,95 & 2,46 \\
\hline \multicolumn{6}{|l|}{ Borda sul } \\
\hline Vochysia tucanorum & 240 & 2,40 & 1,78 & 27,97 & 10,71 \\
\hline Miconia rubiginosa & 1.020 & 10,20 & 4,01 & 9,32 & 7,84 \\
\hline Pterodon pubescens & 360 & 3,60 & 1,33 & 10,93 & 5,29 \\
\hline Ocotea pulchella & 620 & 6,20 & 2,67 & 6,26 & 5,05 \\
\hline Xylopia aromatica & 740 & 7,40 & 4,01 & 3,21 & 4,87 \\
\hline Copaifera langsdorfii & 160 & 1,60 & 2,23 & 7,31 & 3,71 \\
\hline Myrcia splendens & 480 & 4,80 & 2,68 & 3,63 & 3,70 \\
\hline Bauhinia rufa & 420 & 4,20 & 3,12 & 1,22 & 2,84 \\
\hline Virola sebifera & 340 & 3,40 & 2,68 & 1,71 & 2,59 \\
\hline Myrcia guianensis & 320 & 3,20 & 3,12 & 0,78 & 2,36 \\
\hline \multicolumn{6}{|l|}{ Interior sul } \\
\hline Anadenanthera peregrina & 575 & 3,01 & 2,81 & 30,74 & 12,19 \\
\hline Virola sebifera & 2.225 & 11,64 & 1,60 & 2,28 & 5,42 \\
\hline Xylopia aromatica & 1.350 & 7,06 & 3,21 & 6,00 & 5,18 \\
\hline
\end{tabular}


Tabela 2 (continuação)

\begin{tabular}{lrrrrc}
\hline Espécie & \multicolumn{5}{c}{ Parâmetros estruturais } \\
\hline Interior sul & \multicolumn{7}{c}{} \\
\hline Ocotea pulchella & 1.100 & 5,75 & 2,01 & 6,81 & 4,86 \\
Myrcia guianensis & 925 & 4,84 & 3,21 & 3,25 & 3,77 \\
Dalbergia miscolobium & 350 & 1,83 & 0,40 & 8,84 & 3,69 \\
Miconia rubiginosa & 625 & 3,27 & 2,41 & 7,77 & 3,45 \\
Myrcia splendens & 950 & 4,97 & 3,21 & 1,80 & 3,33 \\
Schefflera vinosa & 675 & 3,53 & 3,21 & 2,20 & 2,98 \\
Tocoyena formosa & 575 & 3,01 & 3,21 & 2,28 & 2,61 \\
\hline
\end{tabular}

apresentaram baixos teores de manganês e foram caracterizadas pela presença de Pterodon emarginatus Vogel (P11 com sete indivíduos e P12 com 10). Com base na propriedade centroide e na correlação de Pearson com o lado positivo do eixo 1, num as espécies Amaioua guianensis Aubl. e Tocoyena formosa (Cham. \& Schltdl.) K. Schum., que foram amostradas com respectivamente 27 e 23 indivíduos no interior sul, foram correlacionadas com o interior sul. As espécies que apresentaram maior correlação de Pearson com o lado negativo do eixo 1 foram Aegiphylla lhotzkiana Cham. e Bauhinia rufa (Bong.) Steudel, que apresentaram respectivamente 11 e 62 indivíduos na borda leste.

\section{Discussão}

O levantamento florístico realizado mostrou que o Cerrado sensu stricto em Itirapina apresenta

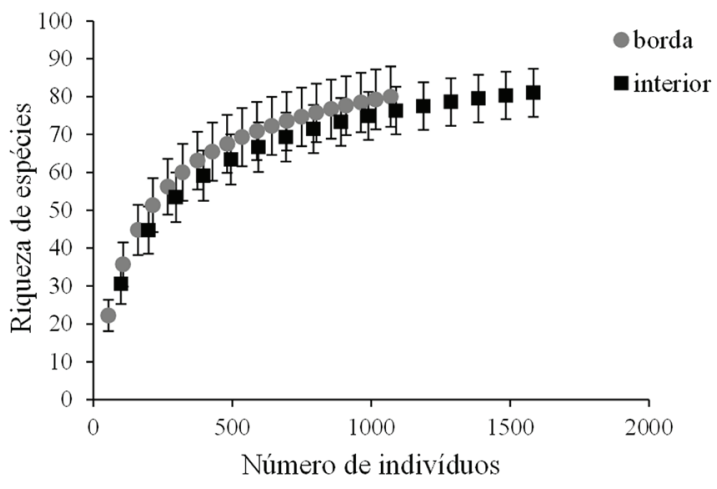

Figura 2. Riqueza de espécies (borda e interior) versus número de indivíduos estimado segundo Mao Tau (Cowell et al. 2004, Mao $\&$ Cowell 2005), Intervalo de confiança $\pm 95 \%$, em um Cerrado sensu stricto em Itirapina, SP, Brasil.

Figure 2. Species richness (edge and interior) versus number of individuals estimated according to Mao Tau (Cowell et al. 2004, Mao \& Cowell 2005), Confidence interval $\pm 95 \%$, in a Cerrado sensu stricto at Itirapina, São Paulo State, Brazil. elevada riqueza de espécies arbustivas e arbóreas ( $\mathrm{n}=120$ espécies) concordando com o que Ratter et al. (2003) encontraram para o Estado de São Paulo: alta riqueza, flora típica e endêmica. Das 120 espécies amostradas, 79 foram encontradas em outras 315 áreas de Cerrado do país, excluindo alguns sítios da Amazônia, listadas por Ratter et al. (2003). Isso indica que o Cerrado sensu stricto em Itirapina possui

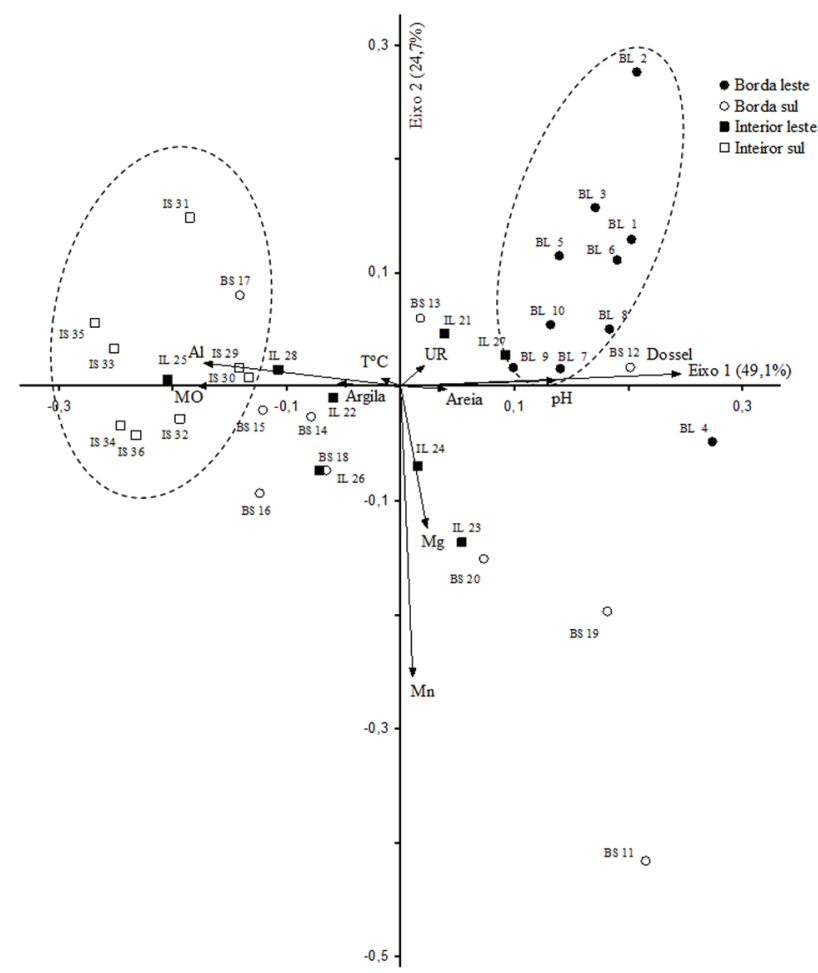

Figura 3. Diagrama de ordenação por PCA (biplot) das variáveis abióticas nas 36 parcelas (1-10 borda leste, 11-20 borda sul, 2128 interior leste, 29-36 interior sul) em um Cerrado sensu stricto localizado em Itirapina, SP, Brasil.

Figure 3, PCA ordination diagram (biplot) of abiotic variables on 36 plots (1-10 East edge, 11-20 South edge 21-28 East interior, 29-36 South interior) in a Cerrado sensu stricto at Itirapina, São Paulo State, Brazil. 
Tablela 3. Similaridade florística entre os quatro ambientes amostrados no Cerrado sensu stricto em Itirapina, SP, Brasil, baseadas no Índice de Chao-Sørensen, Intervalo de confiança $\pm 95 \%$.

Table 3. Floristic similarity among the four environments sampled in the Cerrado sensu stricto at Itirapina, São Paulo State, Brazil, based on Chao-Sorensen Index, confidence interval $\pm 95 \%$.

\begin{tabular}{|c|c|c|c|c|}
\hline & Borda Leste & Borda Sul & Interior Leste & Interior Sul \\
\hline Borda Leste & & $0,55 \pm 0,04$ & $0,77 \pm 0,03$ & $0,68 \pm 0,05$ \\
\hline Borda Sul & & & $0,66 \pm 0,04$ & $0,70 \pm 0,04$ \\
\hline Interior Leste & & & & $0,69 \pm 0,06$ \\
\hline
\end{tabular}

espécies bem representativas de Cerrado apesar de não se localizar na sua área nuclear, considerada mais rica em espécies em relação às áreas periféricas (Castro \& Martins 1999). Apenas três espécies encontradas na área estudada foram indicadas como raras por Ratter et al. (2003), devido à sua presença em apenas uma das 376 áreas comparadas, Eugenia pyriformis Cambess, Leandra solenifera Cogn. e Machaerium brasiliensis Vogel. As famílias Myrtaceae, Fabaceae e Malpighiaceae estão entre as de maior riqueza de espécies em áreas de Cerrado sensu stricto em outras regiões do Brasil, como mostrado por Saporetti et al. (2003) para Estado de Minas Gerais e Borges \& Shepherd (2005) e para o de Mato Grosso.

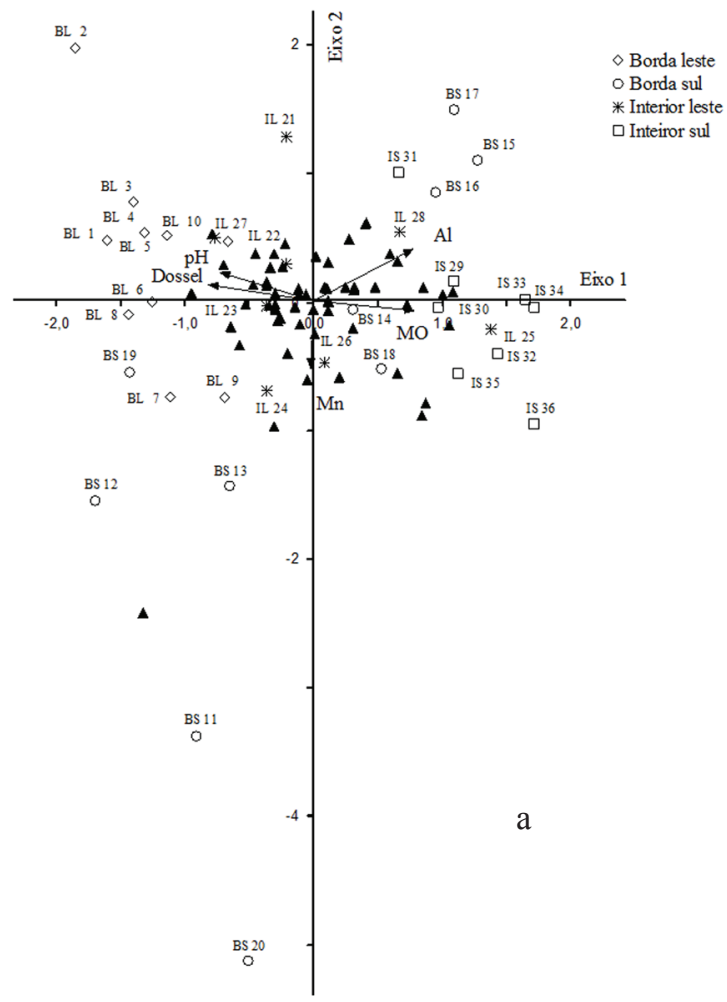

As características microambientais não diferiram entre borda e interior do Cerrado sensu stricto estudado, contrariando os resultados obtidos em comparações similares em estudos de influência de borda em florestas (e.g. Didham \& Lawton, 1999, Gehlhausen et al. 2000, Delgado et al. 2007) e cerradão (Lima-Ribeiro 2008, Hennenberg et al. 2009). Entretanto, as faces leste e sul diferiram significativamente quanto às variáveis microambientais, sendo a face leste, mais ensolarada, apresentando os valores mais elevados de temperatura, PAR e abertura do dossel, e as menores percentagens de umidade relativa. Nosso estudo mostrou que esta diferença influenciou a estrutura e composição da vegetação, assim como afetou a fenologia das espécies naquela área (ver Camargo et al. 2011). A orientação

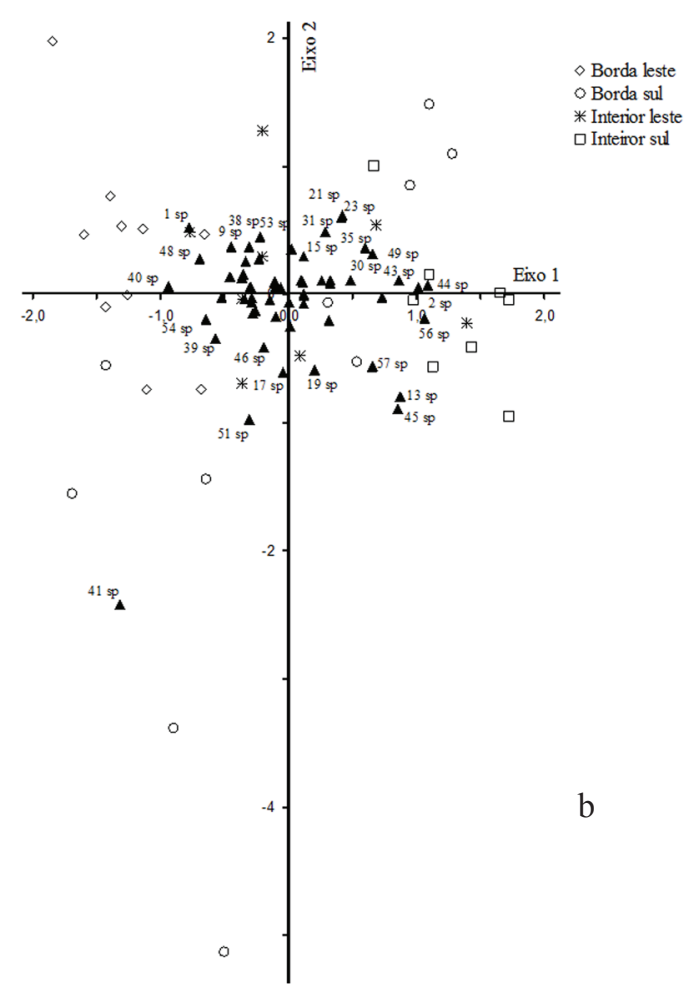

Figura 4. Diagrama de ordenação (CCA); a: unidades amostrais e variáveis microambientais, b: espécies mais abundantes amostradas em Cerrado sensu stricto, Itirapina, SP, Brasil. Espécies e as respectivas correlações de Pearson com o eixo estão indicadas na tabela 1.

Figure 4. CCA ordination diagram; a: sampling units and microenvironmental variables b: most abundant species sampled in a Cerrado sensu stricto at Itirapina, São Paulo State, Brazil. Species and their correlation with Pearson axis are indicated in the Table 1. 


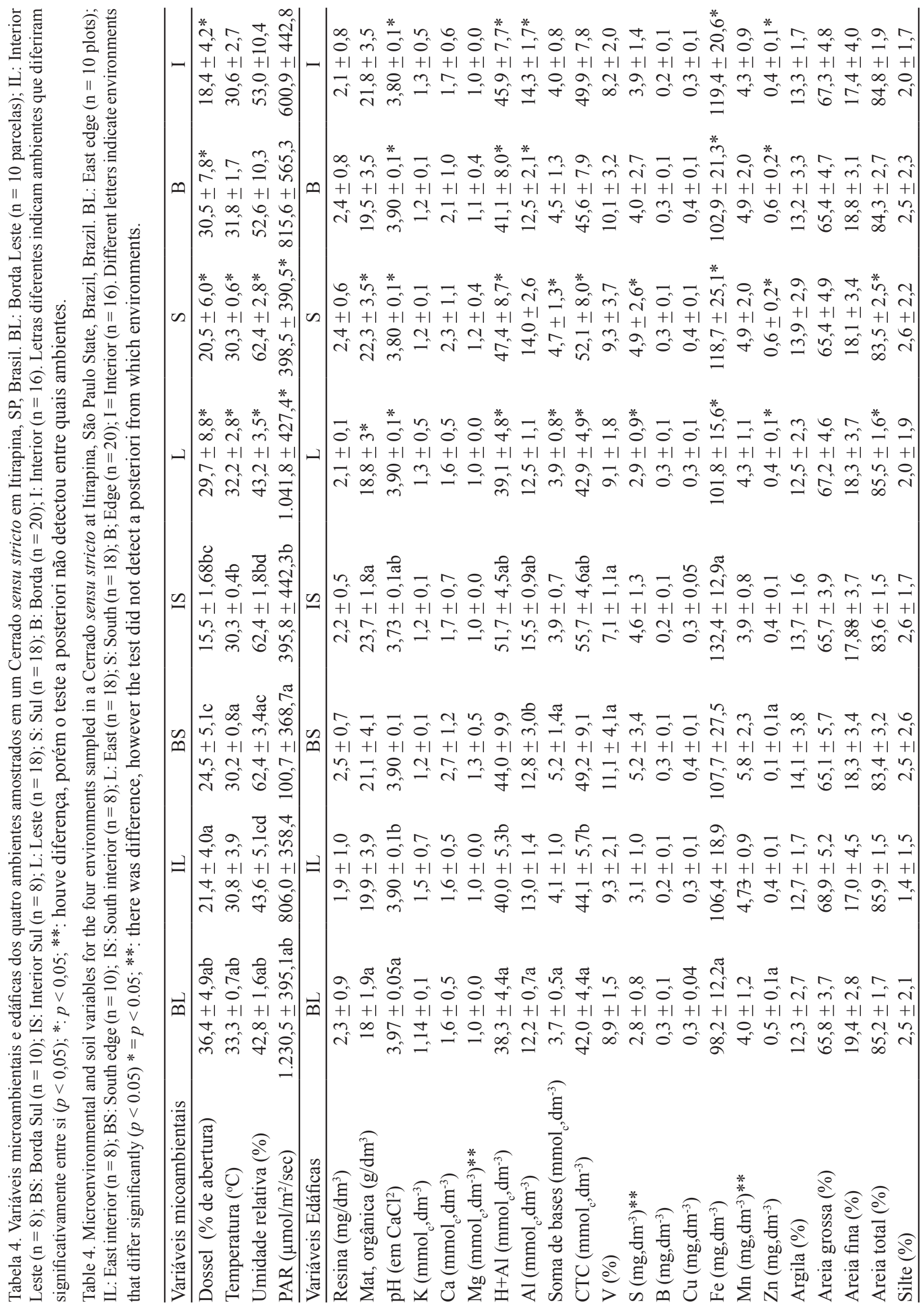


cardinal desempenha um papel fundamental em variáveis como temperatura do ar, do solo e umidade, alcançando os valores mais extremos na face que recebe radiação solar direta (Chen et al. 1993, 1995).

Dentre os parâmetros estruturais avaliados, a densidade absoluta foi o único que diferiu significativamente entre borda e interior do Cerrado. Tanto as variáveis edáficas quanto as variáveis microambientais condicionaram o agrupamento das parcelas e das espécies em função das faces leste e sul, sugerindo que a orientação das faces e as condições microambentais associadas devem influenciar a heterogeneidade da vegetação no Cerrado estudado.

A face leste apresenta uma fisionomia mais aberta, característica de Cerrado sensu stricto típico (sensu Ribeiro \& Walter 2008), onde os maiores indivíduos não passam de $8 \mathrm{~m}$ de altura e as copas das árvores não formam um dossel contínuo, possibilitando a entrada de luz até o estrato herbáceo-subarbustivo. Por outro lado, a face sul exibe uma fisionomia fechada, com indivíduos até $12 \mathrm{~m}$ de altura e dossel contínuo, caracterizando um Cerrado sensu stricto denso (sensu Ribeiro \& Walter 2008). A concentração de certos nutrientes no solo, como por exemplo, o Mn na área de estudo, pode estar determinando o adensamento do Cerrado sensu stricto estudado, definindo a transição entre a fitofisionomia típica na face leste para a densa na face sul. Segundo Pinto et al. (2009), a concentração de nutrientes no solo pode influenciar a estrutura da vegetação do Cerrado. Os solos de Cerrado são empobrecidos quanto à presença de nutrientes como $\mathrm{Mn}, \mathrm{Co}, \mathrm{Ni}, \mathrm{Cu}$ e $\mathrm{Zn}$ (Marques et al. 2004). Especificamente, no caso da borda sul, a maior quantidade de manganês foi positivamente correlacionada à presença de indivíduos de Virola sebifera, Vochysia tucanorum e Pterodon emarginatus, mais altos e com maior área basal nesse ambiente. A quantidade de determinados nutrientes como N, $\mathrm{C}$ e $\mathrm{P}$, a disponibilidade de matéria orgânica e o pH têm sido associados à presença de espécies nativas no México (Toledo-Aceves \& García-Oliva 2008) e Canadá (Marchand \& Houle 2005).

Diferenças na riqueza de espécies nos Cerrados são relacionadas à existência de sub-regiões floristicamente distintas caracterizadas por espécies melhor adaptadas ao amplo espectro de condições ambientais nas quais esse ecossistema ocorre no Brasil (Durigan et al. 2002). Diferentes estudos apontam que as plantas são distribuídas de forma não aleatória em ambientes tropicais, devido à heterogeneidade do habitat, de forma que as diferenças florísticas são melhor explicadas pelo determinismo ambiental, quando comparado à limitação de dispersão (Clark et al. 1998, 1999, Tuomisto et al. 2003). Para a floresta tropical úmida, em trabalhos que enfocam somente a variação ambiental, os fatores que mais comumente afetam a distribuição florística tanto em escala local $\left(<1 \mathrm{~km}^{2}\right)$ quanto em escala média $\left(1-10 \mathrm{~km}^{2}\right)$ incluem a topografia e características edáficas como fertilidade do solo, textura e drenagem (Vormisto et al. 2000, Harms et al. 2001, Vormisto et al. 2004, Valencia et al. 2004).

Para áreas de Cerrado não estão definidos quais os fatores que atuam na determinação dos diferentes tipos de fisionomias, visto que enquanto alguns trabalhos apontam a disponibilidade de nutrientes no solo como fator determinante, outros não confirmam tal relação (Furley \& Ratter 1988, Ribeiro \& Walter 1998, Ruggiero et al. 2002, Ratter et al. 2003, Pinto et al. 2009). Para esse tipo vegetacional são citados ainda fatores como sazonalidade climática, nível do lençol freático, drenagem do solo e influência de queimadas (Oliveira-Filho et al. 1989, Oliveira-Filho $\&$ Ratter 2002).

A influência de borda não foi constatada no Cerrado estudado. Entretanto, demonstramos que a face de exposição influenciou a composição e estrutura da comunidade arbustivo-arbórea. Embora solos sejam tradicionalmente analisados em estudos sobre a composição, estrutura e distribuição dos cerrados, nossos resultados demonstraram a importância da orientação cardinal, e das variações microambientais associadas à face de exposição, além da composição de nutrientes, como fatores que influenciam, em escala local, a fisionomia da vegetação do Cerrado. A orientação cardinal não tem sido considerada em estudos vegetacionais e tem sido negligenciada em estudos de influência de borda (Chen et al. 1995), apesar da sua influência já ter sido sugerida para regiões temperadas (Wales 1972), na distribuição de briófitas (Hylander 2005) e constatado seu efeito na fenologia reprodutiva de espécies de Cerrado (Camargo et al. 2011). As informações obtidas no presente estudo fornecem subsídios novos e importantes a serem considerados em medidas de recuperação de áreas de Cerrado. Destacamos o entendimento das variações estruturais e florísticas relacionadas a presença de bordas e, principalmente, a importância da orientação cardinal na estrutura e dinâmica da vegetação, atributos que influenciam a composição em espécies e funções ecossistêmicas associadas a restauração ecológica (Shackelford et al. 2013). 


\section{Agradecimentos}

Agradecemos ao Instituto Arruda Botelho (IAB) por permitir a realização deste trabalho na Fazenda São José. À Fundação de Amparo Pesquisa do Estado de São Paulo - FAPESP(07/59779-6), pelo apoio financeiro. A autora P.R. teve bolsa de doutorado do CNPq, M.G.G.C. bolsa de mestrado e doutorado da FAPESP (05/57740-0 e 10/01762-3), L.P.C.M. é bolsista de produtividade em pesquisa do CNPq. Este trabalho é uma contribuição do Laboratório de Fenologia e do Grupo de Fenologia e Dispersão de Sementes da Universidade Estadual Paulista em Rio Claro.

\section{Literatura citada}

APG III. 2009. An update of the Angiosperm Phylogeny Group classification for the orders and families of flowering plants. APG III. Botanical Journal of the Linnaean Society 161: 105-121.

Araújo, G.M. \& Haridasan, M. 1988.A comparison of the nutrient status of two forests on dystrophic and mesotrophic soils in the cerrado region of central Brazil. Communications in Soil Science and Plant Analysis 19: 1075-1089.

Assunção, S.L. \& Felfili, J.M. 2004. Fitossociologia de um fragmento de cerrado sensu stricto na APA do Paranoá, DF, Brasil. Acta Botanica Brasilica 18: 903-909.

Baker, J., French, K. \& Whelan, R.J. 2002. The edge effect and ecotonal species: bird communities across a natural edge in southeastern Australia. Ecology 83: 3042-3059.

Balduino, A.P.C., Souza, A.L., Meira Neto, J.A., Silva, A.F. \& Silva Júnior, M.C. 2005. Fitossociologia e análise comparativa da composição florística do cerrado da flora de Paraopeba - MG. Revista Árvore 29: 25-34.

Barbosa, R.I. \& Fearnside, P.M. 2004. Wood density of trees in open savannas of the Brazilian Amazon. Forest Ecology and Management 199: 115-123.

Bittencourt, M.D. \& Mendonça, R.R. 2004. Introdução. In: Viabilidade de Conservação dos Remanescentes de Cerrado (M.D. Bittencourt \& R.R. Mendonça eds.). Annablume, FAPESP, São Paulo. pp. 11-15.

Borges, H.B. \& Shepherd, G.J. 2005. Flora e Estrutura do estrato lenhoso numa comunidade de Cerrado em Santo Antônio do Leverger, MT, Brasil. Revista Brasileira de Botânica 28: 61-74.

Camargo, M.G.G., Souza, R.M., Reys, P. \& Morellato, L.P.C. 2011 Effects of environmental conditions associated to the cardinal orientation on the reproductive phenology of the cerrado savanna tree Xylopia aromatica (Annonaceae). Anais da Academia Brasileira de Ciências 83: 1007-1019.
Castro, A.A.J.F. \& Martins F.R. 1999. Cerrados do Brasil e do Nordeste: caracterização, área de ocupação e considerações sobre a sua fitodiversidade. Pesquisa em Foco 7: 147-178.

Chao, A., Chazdon, R.L., Colwell, R.K. \& Shen, T.J. 2005. A new statistical approach for assessing similarity of species composition with incidence and abundance data. Ecology Letters 8: 148-159.

Chao, A., Chazdon, R.L., Colwell, R.K. \& Shen, T.J. 2006. Abundance-based similarity indices and their estimation when there are unseen species in samples. Biometrics 62: 361-371.

Chen, J., Franklin, J.F. \& Spies, T.A. 1993. Contrasting microclimates among clearcut, edge, and interior old-growth Douglas-fir forest. Agricultural and Forest Meteorology 63: 219-237.

Chen, J., Franklin, J.F. \& Spies, T.A. 1995. Growingseason microclimatic gradients from clearcut edges into old-growth douglas-fir forests. Ecological Aplications 5: 74-86.

Clark, D.B., Clark, D.A. \& Read, J.M. 1998. Edaphic variation and the mesoscale distribution of tree species in a neotropical rain forest. Journal of Ecology 86: 101-112.

Colwell, R.K., Mao, C.X. \& Chang, E.J. 2004. Interpolating, extrapolating and comparing incidencebasedspecies accumulation curves. Ecology 85: 2717-2727.

Colwell, R. 2009. Estimates: Statistical estimation of species richness and shared species from samples. Version 8.2. http://viceroy.eeb.uconn.edu/Estimates (acesso em 26. 04.2012).

Coutinho, L.M. 1978. O conceito de cerrado. Revista Brasileira de Botânica 1: 17-23.

Coutinho, L.M. 2006. O conceito de bioma. Revista Brasileira de Botânica. 20: 1-11.

Dangerfield, J.M., Pik, A. J., Britton, D., Holmes, A., Gillings, M., Oliver, I., Briscoe, D. \& Beattie, A.J. 2003. Patterns of invertebrate biodiversity across a natural edge. Austral Ecology 28: 237-236.

Delgado, J.D., Arroyo, N.L., Arévalo, J.R. \& FernndézPalacios J.M. 2007. Edge effects of roads on temperature, light, canopy cover, and canopy height in laurel and pine forests (Tenerife, Canary Islands). Landscape and Urban Planning 81: 328-340.

Didham, R.K. \& Lawton, J.H. 1999. Edge structure determines the magnitude of changes in microclimate and vegetation structure in tropical forest fragments. Biotropica 31: 17-30.

Durigan, G., Nishikawa, D.L.L., Rocha, E., Silveira, E.L., Pulitano, F.M., Regalada, L.B., Carvalhaes, M.A., Paranaguá, P.A. \& Ranieri, V.E.L. 2002. Caracterização de dois estratos da vegetação em uma área de cerrado no município de Brotas, SP, Brasil. Acta Botanica Brasilica 16: 251-262. 
Durigan, G., Siqueira, M.F., Franco, G.A.D.C. \& Ratter, J.A. 2006. Seleção de fragmentos prioritários para a criação de unidades de conservação do cerrado no estado de São Paulo. Revista do Instituto Florestal 18: 23-37.

Felfili, J.M. \& Silva Junior, M.C. 1992. Florist composition, phytosociology and comparison of cerrado and gallery forest at Fazenda Água Limpa, Federal District, Brazil. In: P.A. Furley, J.A. Proctor \& J.A. Ratter (eds.). Nature and dynamics of forest savanna boundaries. Chapman \& Hall, London, pp. 393-415.

Felfili, J.M., Silva Júnior, M.C., Sevilha, A.C., Fagg, C.W., Walter, B.M.T., Nogueira, P.E. \& Rezende, A.V. 2004. Diversity, floristic and structural patterns of cerrado vegetation in Central Brazil. Plant Ecology 175: 37-46.

Fonseca, M.S. \& Silva Júnior, M.C. 2004. Fitossociologia e similaridade florística em trechos de Cerrado sentido restrito em interflúvio e em vale no Jardim Botânico de Brasília. Acta Botanica Brasilica 18: 19-29.

Frazer, G.W., Canham, C.D. \& Lertxman, K.P. 1999. Gap Light Analyzer (GLA) version 2.0: Imaging software to extract canopy structure and gap light transmission indices from true-colour fisheye photographs, users manual and program documentation. Simon Fraser University, Burnaby, British Columbia, Canada and the Institute of Ecosystem Studies, Millbrook, New York, USA.

Furley, P.A. \& Ratter, J.A. 1988. Soil resources and plant communities of the central Brazilian cerrado and their development. Journal of Biogeography 15: 97-108.

Gehlhausen, S.M., Schwartz, M.W. \& Augspurger, C.K. 2000. Vegetation and microclimatic edge effects in two mixed-mesophyticforest fragments. Plant Ecology 147: 21-35.

Gottsberger, G. \& Silberbauer-Gottsberger, I. 2006. Life in the Cerrado: A South American Tropical Seasonal Ecosystem, Pollination and Seed Dispersal, Reta Verlag, Ulm.

Haridasan, M. 2008. Nutrição mineral de plantas nativas do cerrado. Revista Brasileira de Fisiologia. 12: 54-64.

Harms, K.E., Condit, R., Hubbell, S.P. \& Foster, R.B. 2001. Habitat associations of trees and shrubs in a 50-ha neotropical forest plot. Journal of Ecology 89: 947-959.

Hennenberg, K.J.D., Goetze, J.R., Szarzynski, B., Orthmann, B.R., Reineking, Steinke, I. Porembski, S. 2008. Detection of seasonal variability in microclimatic borders and ecotones between forest and savanna. Basic and Applied Ecology 9: 275-285.

Honnay, O.; Verheyen, K. \& Hermy, M. 2002. Permeability of ancient forest edges for weedy plant species invasion. Forest Ecology and Management 161: 109-122.

Hylander, K. 2005. Aspect modifies the magnitude of edge effects on bryophyte growth in boreal forests. Journal of Applied Ecology 42: 518-525.
Jardim, A.V.F. \& Batalha, M.A. 2009. Dispersal syndromes related to edge distance in cerrado sensu stricto fragments of central-western Brazil. Brazilian Archives of Biology and Technology 52: 1167-1177.

Kapos, V., Wandelli, E., Camargo, J.L. \& Ganade, G. 1997. Edge-related changes in environmental and plant responses due to forest fragmentation in Central Amazonia. In: W.F. Laurance \& R.O. Bierregaard (eds.). Tropical forest remnants. Chicago University Press, Chicago, pp. 33-44.

Klink,C.A. \& Machado, R.B. 2005."A conservação do Cerrado brasileiro". Megadiversidade 1: 147-155.

Köppen, W. 1948. Climatologia. Fondo de Cultura Económica. México.

Kronka, F.J.N.; Nalon, M.A.; Matsukuma, C.K. 2005. Inventário florestal da vegetação natural do Estado de São Paulo. SEMA, Instituto Florestal do Estado de São Paulo, Imprensa Oficial, São Paulo.

Laurance, W.F. 2008. Theory meets reality: How habitat fragmentation research has transcended island biogeographic theory. Biological Conservation 141: 1731-1744.

Legendre, P. \& Legendre, L. 1998. Numerical Ecology. 2nd. English edition. Amsterdam: Elsevier Science BV.

Lima-Ribeiro, M.S. 2008. Efeitos de borda sobre a vegetação e estruturação populacional em fragmentos de cerradão no sudeste goiano, Brasil. Acta Botanica Brasilica 22: 535-545.

Marchand, P. \& Houle, G. 2006. Spatial patterns of plant species richness along a forest edge: what are their determinants? Forest Ecology and Management 223: 113-124.

Marques J.J., Schulze, D.G., Curi, N. \& Mertzman, S.A. 2004.Trace element geochemistry in Brazilian Cerrado soils. Geoderma, 121: 31-43.

Marris, E. 2005. The forgotten ecosystem. Nature: 437: 944-945.

Mccune, B. \& Mefford, M.J. 2000. PCORD. Multivariate analysis of ecological data, version 6.0. MjM Software Desing, Oregon Glaneden Beach.

Metzger, J.P. \& Rodrigues, R.R. 2008. Mapas-síntese. In: Diretrizes para a conservação e restauração da biodiversidade no Estado de São Paulo. Secretaria do Meio Ambiente do Governo do Estado de São Paulo \& Fundação de Amparo à Pesquisa do Estado de São Paulo, São Paulo, pp. 132-139.

Morellato, L.P.C., Gressler, E., \& Camargo, M.G.G., 2013. A review of plant phenology in South and Central America. In: M.D. Schwartz (ed.). Phenology: An Integrative Environmental Science. Springer, New York.

Müeller-Dombois, D. \& Ellenberg, H. 1974. Aims and Methods of Vegetation Ecology. John Wiley \& Sons, New York. 
Murcia, C. 1995. Edge effects in fragmented forests: implications for conservation. Tree 10: 58-62.

Myers, N., Mittermeier, R.A., Mittermeier, C.G., Fonseca, G.A.B. \& Kent, J. 2000. Biodiversity hotspots for conservation priorities. Nature. 403: 853-858.

Nettesheim, F.C., Carvalho, D.C., Fonseca, C.C., Nunes, R.S., Cavalcanti, D.M., Gabriel, M.M. \& Menezes, L.F. 2010. Estrutura e florística do estrato arbóreo no cerrado sensu stricto de Buritis, Minas Gerais, Brasil. Rodriguesia 61: 731-747.

Pinto, J.R., Lenza, E. \& Pinto, A.S. 2009. Composição florística e estrutura da vegetação arbustivo-arbórea em um cerrado rupestre, Cocalzinho de Goiás, Goiás. Revista Brasileira de Botânica 32: 1-10.

Oliveira Filho, A.T. \& Ratter, J.A. 2002. Vegetation physiognomies and woody flora of the cerrado biome. In: P.S. Oliveira \& R.J. Marquis (eds.). The cerrados of Brazil. Ecology an natural history of a Neotropical savanna. Columbia University Press, New York, pp. 91-120.

Oliveira Filho, A.T., Shepherd, G.J., Martins, F.R. \& Stubblebine, W.H. 1989. Environmental factors affecting physiognomic and floristic variation in an area of cerrado in Central Brazil. Journal of Tropical Ecology 5: 413-431.

Pivello, V.R., Carvalho, V.M.C., Lopes, P.F., Peccinini, A.A. \& Rosso, S. 1999. Abundance and distribution of native and alien grasses in a "cerrado" (Brazilian savanna) Biological Reserve. Biotropica 31:71-82.

Ratter, J.A., Bridgewater, S. \& Ribeiro, J.F. 2003. Analysis of the floristic composition of the Brazilian cerrado vegetation III: Comparison of the woody vegetation of 376 areas. Edinburgh Journal of Botany 60: 57-109.

Raij, B. van. 1983. Avaliação da fertilidade do solo. Editora Franciscana (LAFRAME), Piracicaba.

Reatto, A., Correia J.R. \& Spera, S.T. 1998. Solos do Bioma Cerrado: aspectos pedológicos. In: S.M. Sano \& S.P. Almeida (eds.). Cerrado: ambiente e flora. EmbrapaCPAC, Planaltina, DF, pp. 47-86.

Rezende, A.V., Vale, A.T., Sanquetta, C.R., Filho, A.F. \& Felfili, J.M. 2006. Comparação de modelos matemáticos para estimativa do volume, biomassa e estoque de carbono da vegetação lenhosa de um cerrado sensu stricto em Brasília, DF. Scientia Florestalis 71: 65-76.

Ribeiro, S.C., Fehrmann, L., Soares, C.P.B., Jacovine, L.A.G., Kleinn, C. \& Gaspar R.O. 2011. Above- and belowground biomass in a Brazilian Cerrado. Forest Ecology and Management 262: 491-499.
Ribeiro, J.F. \& Walter, B.M.T. 2008. As principais fitofisionomias do Bioma Cerrado. In: Cerrado: ecologia e flora (S.M. Sano, S.P. Almeida \& J.F. Ribeiro, eds.). Embrapa Cerrados, Planaltina. pp. 151-212.

Ruggiero, P.G.C., Batalha, M.A., Pivello, V.R. \& Meirelles, S.T. 2002. Soil vegetation relationships in cerrado (Brazilian savanna) and semideciduous forest, Southeastern Brazil. Plant Ecology 160:1-16.

Shackelford N., Hobbs R.J., Burgar J.M., Erickson T.E., Fontaine J.B., Laliberté E., Ramalho C.E., Perring M.P. \& Standish R.J. 2013. Primed for Change: Developing Ecological Restoration for the 21st Century. Restoration Ecology 21: 297-304.

Shepherd, G.J. 2010. Fitopac 2.1 - Campinas, Departamento de Botânica, Universidade Estadual de Campinas.

Siqueira, M.F. \& Durigan, G. 2007. Modelagem da distribuição geográfica de espécies lenhosas de cerrado no Estado de São Paulo. Revista Brasileira de Botânica 30: 233-243.

Ter-Braak, C.J.F. 1987. The analysis of vegetationenvironment relationships by canonical correspondence analysis. Plant Ecology, 69: 69-77.

Toledo-Aceves, T. \& García-Oliva, F. 2008. Effects of forest-pasture edge on $\mathrm{C}, \mathrm{N}$ and Passociated with Caesalpinia eriostachys, a dominant tree species in a tropical deciduous forest in Mexico. Ecological Research 23: 271-280.

Tuomisto, H., Ruokolainen, K. \& Yli-Halla, M. 2003. Dispersal, Environment, and Floristic Variation of Western Amazonian Forests. Science 299: 241-244.

Valencia, R., Foster, R.B., Villa, G., Condit, R., Svenning, J.C., Hernández, C.,Romoleroux K., Losos, E., Magard, E. \& Balslev, H. 2004. Tree species distributions and local habitat variation in the Amazon: large forest plot in eastern Ecuador. Journal of Ecology 92: 214-229.

Vormisto, J., Phillips, O. L., Ruokolainen, K. \& Vásquez, R. 2000. A comparison of fine-scale distribution patterns of four plant groups in an Amazonia rain forest. Ecography 23: 349-359.

Vormisto, J., Tuomisto, H. \& Oksanen, J. 2004. Palm distribution patterns in Amazonian rainforests: what is the role of topographic variation. Journal of Vegetation Science 15: 485-494.

Wales, B.A. 1972. Vegetation analysis of north and south edges in a mature oak-hickory forest. Ecological Monographs 42: 451-471.

Zar, H.J. 1999. Biostatistical analysis. Prentice-Hall, New Jersey. 